\title{
Codeword Stabilized Quantum Codes
}

\author{
Andrew Cross, Graeme Smith, John A. Smolin and Bei Zeng
}

\begin{abstract}
We present a unifying approach to quantum error correcting code design that encompasses additive (stabilizer) codes, as well as all known examples of nonadditive codes with good parameters. We use this framework to generate new codes with superior parameters to any previously known. In particular, we find $((10,18,3)),((10,20,3))$ and $((11,48,3))$ codes.
\end{abstract}

Index Terms-quantum error correction, nonadditive codes, stabilizer codes

\section{INTRODUCTION}

Quantum computers hold the promise of the efficient solution of problems, such as factoring [1] and simulation of quantum systems [2], [3], [4] that are generally believed to be intractable on a classical computer. Furthermore, as the processor size in state-of-the-art computers continues to scale down and performance begins to be limited by dissipative effects in logical processing, it has become increasingly clear that considering the quantum nature of the components of a classical computer will be essential in the not-too-distantfuture. In both of these scenarios-constructing a working quantum computer, or simply continuing to improve the performance of classical computers-quantum error correcting codes and ideas from quantum fault-tolerance [5] will be essential elements in the future computer engineer's toolbox.

Stabilizer codes are an important class of quantum codes developed in [6], [7], and are the quantum analogues of classical additive codes. An $[n, k]$ stabilizer code encodes $k$ logical qubits into $n$ physical qubits, and is described by an abelian subgroup, $S$, of the Pauli group with size $|S|=2^{n-k}$. The codespace is the set of simultaneous eigenvectors of $S$ with eigenvalue 1 . There is a rich theory of stabilizer codes, and a thorough understanding of their properties.

Nevertheless, such codes are strictly suboptimal in some settings - there exist nonadditive codes which encode a larger logical space than possible with a stabilizer code of the same length and capable of tolerating the same number of errors. There are only a handful of such examples [8], [9], [10], and their constructions have proceeded in an ad hoc fashion, each code working for seemingly different reasons.

In the following we present a framework for code design that includes as special cases stabilizer codes as well as all known nonadditive codes with good parameters. Our codes are fully described by two objects: a single stabilizer state $|S\rangle$, and a classical code that generates the basis vectors of

Andrew Cross is with the Department of Electrical Engineering, Massachusetts Institute of Technology, Cambridge MA 02139, USA

Andrew Cross, Graeme Smith and John Smolin are with IBM T.J. Watson Research Center, Yorktown Heights, NY 10598, USA

Bei Zeng is with the Department of Physics, Massachusetts Institute of Technology, Cambridge, MA 02139, USA

GS was partially supported by the UK Engineering and Physical Sciences Research Council, JAS was supported by ARO contract DAAD19-01-C-0056. our code from $|S\rangle$. The stabilizer is chosen such that it maps all Pauli errors onto only $Z$ errors, though this may increase their weight. In this way we map the problem of finding a quantum code to that of finding a classical code that corrects an unusual error model. We have thus unified stabilizer and nonadditive codes and rendered both in a form that gives insight into the classical nature of quantum error-correction.

Our approach is related to the description of nonadditive codes given in [11] in terms of Boolean functions, and in particular our codeword operators, codeword stabilizer, and effective classical errors correspond, respectively, to a Boolean function $f$, a matrix $A_{f}$, and the "Zset" in the language of that work. Their approach is essentially dual to ours-in the language we use here it amounts to first choosing a classical code and trying to design a stabilizer state whose induced error model is corrected by the chosen code. From this perspective, the approach of [11] seems somewhat unnatural, which is perhaps the reason it has not proved useful for finding new codes. Both approaches are closely related to the work of [12].

We describe codes on $n$ qubits that encode $K$ dimensions with distance $d$ (traditionally written $((n, K, d)))$. In this framework we find the original nonadditive $((5,6,2))$ code of [8] and the family it generates, the simple family of distance 2 codes found in [9], the $((9,12,3))$ code of [10], as well as new $((10,18,3)),((10,20,3))$ and $((11,48,3))$ codes.

\section{General construction And Properties}

An $((n, K))$ code will be described by two objects- $S$, a $2^{n}$ element abelian subgroup of the Pauli group not containing minus the identity, which we call the word stabilizer, together with a family of $K n$-qubit Pauli elements, $W=\left\{w_{l}\right\}_{l=1}^{K}$, which we call the word operators. There is a unique state $|S\rangle$ stabilized by $S$, i.e. $|S\rangle$ satisfies $s|S\rangle=|S\rangle$ for all $s \in S$. Our code will be spanned by basis vectors of the form

$$
\left|w_{l}\right\rangle \equiv w_{l}|S\rangle
$$

Since the code vectors should all be different, at most one $w_{l}$ can be in $S$. Typically we will choose $w_{1}=I$ and later we will prove this can be done without loss of generality. Note that $\left|w_{l}\right\rangle$ is an eigenvector of all $s \in S$ with eigenvalue $\lambda_{s}= \pm 1$, but $\left|w_{l}\right\rangle$ is not stabilized by $S$ unless $w_{l} \in S$. Each $\left|w_{l}\right\rangle$ is stabilized by a different stabilizer $w_{l} S w_{l}^{\dagger}$.

We would now like to understand the error correction capabilities of such a codeword stabilized (CWS) code. An $((n, K, d))$ code is an $((n, K))$ code capable of detecting Pauli errors of weight up to $d-1$, but not $d$, and is said to have minimum distance $d$. A distance $d$ code can also be used to correct errors up to weight $\lfloor(d-1) / 2\rfloor$. The conditions for error correction were found in [13], [14]. The error correction conditions for a general code with basis vectors $\left|w_{l}\right\rangle$ are that, 
in order to detect errors from a set $\mathcal{E}$, it is necessary and sufficient to have

$$
\left\langle c_{i}|E| c_{j}\right\rangle=c_{E} \delta_{i j}
$$

for all $E \in \mathcal{E}$. For a code of the form described above, this becomes

$$
\left\langle S\left|w_{i}^{\dagger} E w_{j}\right| S\right\rangle=c_{E} \delta_{i j} .
$$

This leads to the necessary and sufficient condition for detecting errors from $\mathcal{E}$ that for all $E \in \mathcal{E}$

$$
\forall i \neq j w_{i}^{\dagger} E w_{j} \notin \pm S
$$

and

$$
\begin{aligned}
& \left(\forall i w_{i}^{\dagger} E w_{i} \notin \pm S\right) \text { or } \\
& \left(\forall i w_{i}^{\dagger} E w_{i} \in S\right) \text { or } \\
& \left(\forall i w_{i}^{\dagger} E w_{i} \in-S\right)
\end{aligned}
$$

Eq. (4) is the condition that two codewords should not be confused after an error, while the final three conditions express that each error must either be detected (Eq. (5)), or the code must be "immune" to it-i.e. the code is degenerate.

Theorem 1 An $((n, K))$ codeword stabilized code with word operators $W=\left\{w_{l}\right\}_{l=1}^{K}$ and codeword stabilizer $S$ is locally Clifford-equivalent to a codeword stabilized code with word operators $w_{l}^{\prime}=Z^{\mathbf{c}_{l}}$ and codeword stabilizer $S^{\prime}$ generated by

$$
S_{l}^{\prime}=X_{l} Z^{\mathbf{r}_{l}} .
$$

In other words, any CWS code is locally equivalent to a CWS code with a graph-state stabilizer and word operators consisting only of $Z s$. The set of $\mathbf{r}_{l}$ s form the adjacency matrix of the graph. Moreover, the word operators can always be chosen to include the identity. We call this standard form.

Proof: First note that $S$ is local-Clifford equivalent to a graph state due to [15], [16], [17]so there is some localClifford unitary $C=\bigotimes_{l=1}^{n} C_{l}$ that maps $S$ to $S^{\prime}$ of the form (8). In the new basis the word operators are $C w_{l} C^{\dagger}=$ $\pm Z^{\mathbf{a}_{l}} X^{\mathbf{b}_{l}}$, and we have

$$
C w_{l} C^{\dagger} \prod_{i}\left(S_{i}^{\prime}\right)^{\left(\mathbf{b}_{l}\right)_{i}}= \pm Z^{\mathbf{c}_{l}},
$$

so that, letting $w_{l}^{\prime}=Z^{\mathbf{c}_{l}}$, we have

$$
\begin{aligned}
Z^{\mathbf{c}_{l}}\left|S^{\prime}\right\rangle & = \pm C w_{l} C^{\dagger} s^{\prime}\left|S^{\prime}\right\rangle \\
& = \pm C w_{l} C^{\dagger}\left|S^{\prime}\right\rangle \\
& = \pm C w_{l}|S\rangle .
\end{aligned}
$$

Since $C$ consists of local Clifford elements, we see that the CWS code defined by $S^{\prime}$ and $w^{\prime}$ is locally Clifford equivalent to the original code.

Finally, to ensure the codeword operators include the identity we can choose $\tilde{W}=\left\{\tilde{w}_{l}=w_{l}^{\prime} w_{1}^{\prime}\right\}$ which always has $\tilde{w}_{1}=$ Identity. This can be seen by commuting the $w_{1}^{\prime}$ through the $E$ in the error-correction conditions which can at worst pick up a sign depending only on $E$. The two conditions

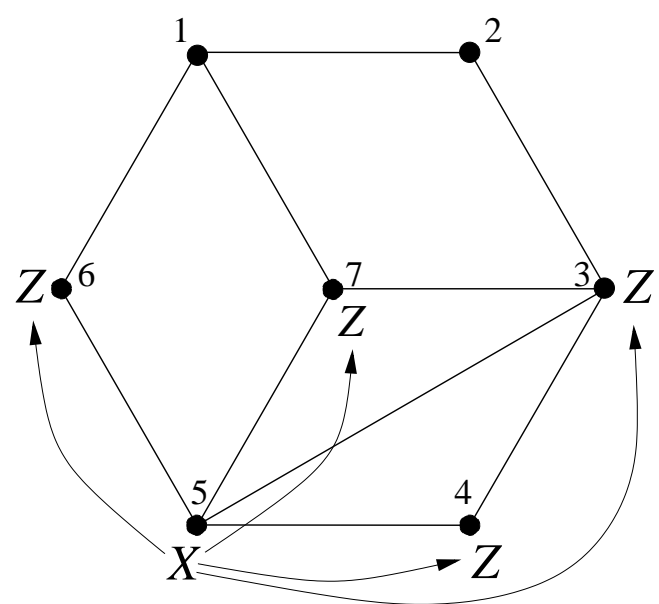

Fig. 1. Example of the induced error on a graph state: The state has stabilizer generators $X Z I I I Z Z, Z X Z I I I I, I Z X Z I I Z, I I Z X Z I I, I I Z Z X Z Z$, $Z I I I Z X I$, and $Z I Z I Z I X$. An $X$ error applied to node 5 in the lower-left is translated by multiplying with the stabilizer element $I I Z Z X Z Z$ and turns into $Z$ errors on the nodes indicated.

with $\pm S$ on the right are insensitive to this and the other two conditions at most change places.

This structure theorem gives rise to the following lemma, which is at the heart of our construction:

Lemma 2 A single qubit Pauli error $Z, X$ or $Y=Z X$ acting on a codeword $w|S\rangle$ of a CWS code in standard form is equivalent up to a sign to another (possibly multi-qubit) error consisting only of $Z s$.

Proof: Let the error $E_{i}$ act only on the $i$ th qubit. If it is a $Z$ error the result is immediate. Otherwise use the fact that $E_{i} w|S\rangle= \pm E_{i} S_{i} w|S\rangle$, and take $S_{i}$ to be the generator having $X$ on bit $i$. Then since $E_{i}=Z_{i}^{\{0,1\}} X_{i}$ the $X$ in $E_{i}$ cancels with the $X$ from $S_{i}$ and we are left with the $Z$ s from $S_{i}$ as well as a $Z_{i}$ if $E_{i}$ was $Z_{i} X_{i}$.

Lemma 2 allows us to construct CWS codes with a satisfying interpretation: $X$ errors on any qubit are "pushed" outwards along the edges of the graph and transformed into $Z$ s. This is illustrated in figure 1 Similarly $Y$ errors are pushed along the edges, but also leave a $Z$ behind at their original locations. Since all errors become $Z$ s, we can think of the error model as classical, albeit consisting of strange multibit errors. We define this translation to classical errors by the function $C l_{S}(E \in \mathcal{E}) \rightarrow\{0,1\}^{n}$ :

$$
C l_{S}\left(E= \pm Z^{\mathbf{v}} X^{\mathbf{u}}\right)=\mathbf{v} \oplus \bigoplus_{l=1}^{n}(\mathbf{u})_{l} \mathbf{r}_{l}
$$

where $\mathbf{r}_{l}$ is the $l$ th row of the stabilizer's adjacency matrix (recall from Eq. (8) $S_{l}=X_{l} Z^{\mathbf{r}_{l}}$ defines $\mathbf{r}_{l}$ ). The codeword operators $w_{l}=Z^{\mathbf{c}_{l}}$ will be chosen to so that the $\mathbf{c}_{l} \mathrm{~s}$ are a classical code for this error model.

Theorem 3 A CWS code in standard form with stabilizer $S$ and codeword operators $\left\{Z^{\mathbf{c}}\right\}_{\mathbf{c} \in C}$ detects errors from $\mathcal{E}$ if and 
only if $C$ detects errors from $C l_{S}(\mathcal{E})$ and in addition we have for each $E$,

$$
\begin{aligned}
C l_{S}(E) & \neq 0 \\
\text { or } \forall i Z^{\mathbf{c}_{i}} E & =E Z^{\mathbf{c}_{i}} \\
\text { or } \forall i Z^{\mathbf{c}_{i}} E & =-E Z^{\mathbf{c}_{i}}
\end{aligned}
$$

Proof: When $i \neq j, w_{i}^{\dagger} E w_{j} \notin \pm S$ is satisfied exactly when $Z^{\mathbf{c}_{i}} E Z^{\mathbf{c}_{j}} \notin \pm S$, which is in turn equivalent to $Z^{\mathbf{c}_{i}} Z^{C l_{S}(E)} Z^{\mathbf{c}_{j}} \notin \pm S$. In standard form, the only element of $S$ without any $X$ is the identity, so that this is satisfied exactly when $\mathbf{c}_{i} \oplus C l_{S}(E) \neq \mathbf{c}_{j}$. This is explicitly the classical errordetection condition.

Similarly, when $i=j$, we must satisfy Eqs. (5), (6) and (7), whose three possibilities translate directly to

$$
\begin{array}{r}
\forall \mathbf{c} Z^{\mathbf{c}} E Z^{\mathbf{c}} \notin \pm S \\
\text { or } \forall \mathbf{c} Z^{\mathbf{c}} E Z^{\mathbf{c}} \in S \\
\text { or } \forall \mathbf{c} Z^{\mathbf{c}} E Z^{\mathbf{c}} \in-S,
\end{array}
$$

Since $Z^{\mathbf{c}}=I$ for the $\mathbf{c}=0$ codeword Eq. (17) is equivalent to $E \notin \pm S$ which gives (14). For the final two equations (18) and (19) we know $E \in \pm S$ which which readily implies the conditions 15) and (16).

Remark A classical code expressed in quantum terms would traditionally comprise computational basis vectors that are eigenstates of $Z$, and therefore the operators mapping one codeword to another would be of the form $X^{\mathbf{c}}$ as these are the only errors that have any effect. It then might seem odd that standard form for CWS codes, the intuition of which is to make everything classical, would employ word operators and effective errors consisting only of $Z \mathrm{~s}$. This choice is arbitrary (one could exchange $Z$ and $X$ and nothing in the formalism would be affected) and is made since the usual form of a graph state stabilizer is to have one $X$ and some number of $Z$ s rather than the reverse. We hope this historical accident does not cause too much confusion going forward.

\section{A. Relation to Stabilizer codes}

The CWS framework includes stabilizer codes, and allows them to be understood in a new way. We now show that any stabilizer code is a CWS code, and give a method for determining if a CWS code is also a stabilizer code.

Theorem 4 An $[n, k]$ stabilizer code with stabilizer generators $S_{1}, \ldots, S_{n-k}$ and logical operations $\bar{X}_{1} \ldots \bar{X}_{k}$ and $\bar{Z}_{1} \ldots \bar{Z}_{k}$, is equivalent to the CWS code defined by

$$
S=\left\langle S_{1} \ldots S_{n-k}, \bar{Z}_{1} \ldots \bar{Z}_{k}\right\rangle
$$

and word operators

$$
w_{\mathbf{v}}=\bar{X}_{1}^{(\mathbf{v})_{1}} \otimes \ldots \otimes \bar{X}_{k}^{(\mathbf{v})_{k}}
$$

where $\mathbf{v}$ is a $k$-bit string.

Proof: To see that this CWS code describes the original code, note that the stabilizer state associated with $S$ is $|\overline{0} \ldots \overline{0}\rangle$, while the codeword generated by $W_{\mathbf{v}}$ acting on $|\overline{0} \ldots \overline{0}\rangle$ is $\left|(\overline{\mathbf{v}})_{1} \ldots(\overline{\mathbf{v}})_{k}\right\rangle$.
Theorem 5 If the word operators of an $((n, K)) C W S$ code are an abelian group $W$ (not containing $-I$ ), then the code is an $\left[n, k=\log _{2} K\right]$ stabilizer code.

Proof: The stabilizer $S$ of the CWS code is a maximal abelian subgroup of the Paulis (not containing $-I$ ) therefore it is isomorphic to the group $S^{\prime}=\left\langle X_{1} \ldots X_{n}\right\rangle$ and the mapping from $S$ to $S^{\prime}$ is a Clifford operation $C$ (not necessarily local). This follows from the definition of the Clifford group as the automorphisms of the Pauli group. Because the automorphism group allows one to achieve any mapping that preserves commutation relations, the map can further be chosen to map $W$ to $W^{\prime}=\left\langle Z_{1} \ldots Z_{k}\right\rangle$. Here we have made use of the facts that all $w \in W$ anticommute with at least one $s \in S$ (which imples $S \cap W=\{I\}$ ) and that $S^{\prime}$ is maximal, which allows us to choose for $W^{\prime}$ any order $K$ group made only of $Z$ s we like (since all products of $X^{\prime}$ 's are in $S^{\prime}$ ). Note this nonlocal Clifford mapping is not the same as the conversion to $Z$ s used in Theorem 1

We can now choose $T^{\prime}, \bar{X}^{\prime}$ and $\bar{Z}^{\prime}$ as follows:

$$
\begin{aligned}
\bar{X}^{\prime} & =W^{\prime}=\left\langle Z_{1} \ldots Z_{k}\right\rangle \\
\bar{Z}^{\prime} & =\left\langle X_{1} \ldots X_{k}\right\rangle \\
T^{\prime} & =\left\langle X_{k+1} \ldots X_{n}\right\rangle
\end{aligned}
$$

The inverse Clifford operation $C^{\dagger}$ maps these to our stabilizer code with stabilizer $T$, and logical operations $\bar{X}=W$ and $\bar{Z}$.

It remains to show this is the same as the CWS code we started with. $T$ is by construction a subgroup of $S\left(T^{\prime}\right.$ is explicitly generated by a subset of the generators of $S^{\prime}$ ) and therefore stabilizes $|S\rangle . T$ also stabilizes all $\bar{x}|S\rangle, \bar{x} \in \bar{X}$, since $T$ and $\bar{X}$ commute. Using $\bar{X}=W$ we see these states are exactly the basis states of the CWS code.

\section{KNOWN EXAMPLES}

We now show that our construction encompasses stabilizer codes and all known nonadditive codes with good parameters.

\section{A. The $[5,1,3]$ code}

The celebrated [5, 1, 3] quantum code [13], [14] can be written as a CWS code using Eqs. (20) and (21) but another way of writing it demonstrates the power of the CWS framework. Take generators corresponding to a ring graph:

$$
S_{i}=Z X Z I I \text { and cyclic shifts. }
$$

This induces effective errors as follows. Letting $|R 5\rangle$ be the graph state corresponding to the unique simultaneous +1 eigenvector of these generators, we have

$$
\begin{aligned}
Z_{i}|\mathrm{R} 5\rangle & =Z_{i}|\mathrm{R} 5\rangle \\
X_{i}|\mathrm{R} 5\rangle & =Z_{i-1} Z_{i+1}|\mathrm{R} 5\rangle \\
Y_{i}|\mathrm{R} 5\rangle & =Z_{i-1} Z_{i} Z_{i+1}|\mathrm{R} 5\rangle
\end{aligned}
$$

where all additions and subtractions are taken modulo 5. The corresponding 15 classical errors are:

$$
\begin{array}{llllll}
Z: & 10000 & 01000 & 00100 & 00010 & 00001 \\
X: & 01001 & 10100 & 01010 & 00101 & 10010 \\
Y: & 11001 & 11100 & 01110 & 00111 & 10011
\end{array}
$$


We then must choose $w_{l}=Z^{\mathbf{c}_{l}}$ where the $\mathbf{c}_{l} \mathrm{~s}$ form a classical code capable of detecting pairs of these errors. Since no pair of these errors produces 11111 the codewords $\mathbf{c}_{0}=00000$ and $\mathbf{c}_{1}=11111$ will serve, and together with the stabilizer 25 completely define the code. Since the $((5,2,3))$ code is known to be unique we need not otherwise check that our construction is equivalent to the traditional presentation of this code. We note also that for $n \geq 7$ a ring code with codeword operators $I$ and $\otimes_{l=1}^{n} Z_{l}$ gives a $[n, 2,3]$ code.

\section{B. The $((5,6,2))$ code}

The first nonadditive quantum code was found in [8], and encodes a six-dimensional space into five qubits with a minimum distance of two. This outperforms the best additive five qubit distance two code, which can have an encoded dimension of at most four. The code was originally found as follows: It was known that the linear programming upper bound was exactly 6 for a blocklength 5 distance 2 code, and in fact it was possible to completely determine what the weight enumerator [18] of a code meeting this bound must be. The authors of [8] then performed a numerical search for such a code, and managed to find one. The structure of the resulting code was mysterious, and generating larger codes in a similar fashion seemed intractable (though [19] showed how to construct a $\left(\left(5+2 l, 2^{2 l+1} 3,2\right)\right)$ code from this code).

As a CWS code the $((5,6,2))$ code of [8] becomes simple. We again use the ring stabilizer (25) and will have to detect the induced errors 27, but since we are seeking a distance-2 code we need only consider single errors rather than pairs. The classical codewords $\mathbf{c}_{l}, l=0 \ldots 5$, are

\section{$\begin{array}{llllll}00000 & 11010 & 01101 & 10110 & 01011 & 10101\end{array}$}

and the code generated by $\left|c^{\mathrm{R} 5}\right\rangle$ and $W_{l}=Z^{\mathrm{c}_{l}}$ is locally Clifford equivalent to the $((5,6,2))$ code of [8]. The $((5+$ $\left.\left.2 l, 2^{2 l+1} 3,2\right)\right)$ codes of [19] are also CWS codes whose graph state is the union of the ring graph and $l$ Bell pair graphs, and whose classical codewords can be derived straightforwardly from the $((5,6,2))$ classical codewords.

\section{The SSW codes}

A family of distance two codes was found in [9], which outperforms the family of [19] for odd blocklengths of eleven or larger. The codes were originally described in terms of their codewords as follows. If $n=1 \bmod 4$, a basis of our code consists of vectors of the form

$$
|\mathbf{x}\rangle+|\overline{\mathbf{x}}\rangle,
$$

where $\mathrm{x}$ ranges over all $\mathrm{n}$-bit vectors of odd weight less than $(n-1) / 2$ and $\overline{\mathbf{x}}$ is the complement of $\mathbf{x}$, while if $n=3$ $\bmod 4$, we let $\mathbf{x}$ range over even weight vectors of weight less than $(n-1) / 2$, leading to an encoded dimension of $2^{n-2}\left(1-\frac{\left(\begin{array}{c}n-1 \\ (n-1) / 2\end{array}\right)}{2^{n-1}}\right)$.

We now show that these are actually CWS codes. Indeed, the codeword stabilizer of this code will be generated by

$$
\left\langle X_{1} Z_{2} \ldots Z_{n}, Z_{1} X_{2}, Z_{1} X_{3}, \ldots, Z_{1} X_{n}\right\rangle
$$

with the corresponding stabilizer state being equivalent to a GHZ state, $\left(|0\rangle|+\rangle^{\otimes n-1}+|1\rangle|-\rangle^{\otimes n-1}\right) / \sqrt{2}$. The codeword operators are simply $W_{\mathbf{x}}=X^{(\mathbf{x})_{1}} Z^{\left((\mathbf{x})_{2}, \ldots(\mathbf{x})_{n}\right)}$ for each allowed $\mathbf{x}$, which can immediately be seen to generate, up to local unitaries, the same codewords as Eq. 29. Putting the stabilizer into standard form, we find that the graph state it describes corresponds to a star graph.

\section{The $((9,12,3))$ code}

The $((9,12,3))$ code of [10] was presented explicitly in the form of a CWS code and, indeed, served as motivation for our studies of the generality of such a construction. Like the $((5,6,2))$ code, the codeword stabilizer is of the form

$$
S_{i}=Z X Z I I I I I I \text { and cyclic shifts }
$$

so that the effective classical error model we must be able to correct are given by Eq. 26). We do this by choosing the the codeword operators to be $w_{l}=Z^{\mathbf{c}_{l}}$, where the $\mathbf{c}_{l} \mathrm{~s}$ are:

$\begin{array}{llll}000000000 & 100100100 & 010001100 & 110101000 \\ 000110001 & 100010101 & 011001010 & 111101110 \\ 001010011 & 101110111 & 011111111 & 111011011 .\end{array}$

\section{NEW CODES}

\section{A. Ring codes: $((10,18,3))$ and $((11,48,3))$}

In light of the excellent performance of ring-stabilizers for CWS codes - the $((5,6,2))$ and $((9,12,3))$ are both of this form-we have studied larger blocklength codes based on this stabilizer. This leads to two further codes which outperform stabilizer codes for blocklengths of 10 and 11 .

The blocklength ten code has a codeword stabilizer generated by $\left\langle Z_{i-1} X_{i} Z_{i+1}\right\rangle$ and has 18 word operators of the form $Z^{\mathbf{c}_{l}}$, with $\mathbf{c}_{l}$ taken from the list

$\begin{array}{lll}0000000000 & 1101001100 & 0011001010 \\ 0000011111 & 0010001001 & 1111100000 \\ 1000111110 & 1100100101 & 0101101101 \\ 0001000110 & 1010010010 & 0100110100 \\ 1001011011 & 1011010001 & 0110111000 \\ 0110110010 & 1110100011 & 0111111011 .\end{array}$

Similarly, our blocklength 11 code has the analogous stabilizer, but now word operators of the form $Z^{\mathbf{d}_{l}}$ with $\mathbf{d}_{l}$ taken from the list

$\begin{array}{llll}00000000000 & 11111011101 & 11100011000 & 00101001001 \\ 11011110111 & 10001100101 & 00100110011 & 01011110001 \\ 01001001110 & 10000011111 & 11110100111 & 00110010101 \\ 00100101010 & 00001100011 & 10110010011 & 00101010000 \\ 11010001101 & 10011000011 & 10111110000 & 01110100001 \\ 01011101000 & 10100110101 & 00010100110 & 00111110110 \\ 10001111100 & 10010100000 & 01100000111 & 01100011110 \\ 01101111101 & 11010010100 & 11000110010 & 01110111000 \\ 10010111001 & 10101010110 & 11110111110 & 01000101101 \\ 00001111010 & 10000001010 & 11111000100 & 01000110100 \\ 10100101001 & 11100000001 & 00011000101 & 01010001011 \\ 11000101011 & 00000011001 & 00011011100 & 01010010010 .\end{array}$

That both of these codes satisfy the required error correction conditions can be shown by the straightforward (if tedious) technique of verifying that the associated classical codes correct the classical noise model induced by the ring stabilizer. 


\section{B. A $((10,20,3))$ Double Ring Code}

We now consider a CWS code with a codeword stabilizer that is not of the ring form. In particular, our stabilizer will correspond to the double ring, with generators

$$
\begin{array}{ll}
S_{1}=X Z I I Z Z I I I I & S_{6}=\text { ZIIIIXZIIZ } \\
S_{2}=Z X Z I I I I I & S_{7}=I Z I I Z X Z I I \\
S_{3}=I Z X Z I I I Z I I & S_{8}=I I Z I I Z X Z I \\
S_{4}=I I Z X Z I I I I & S_{9}=I I I Z I I Z X Z \\
S_{5}=Z I I Z X I I I I & S_{10}=I I I I Z I I Z X .
\end{array}
$$

This leads to a $|S\rangle$ that is a $[10,0,4]$ stabilizer state. Our codewords are then of the form $Z^{\mathbf{c}_{l}}|S\rangle$ with $\mathbf{c}_{l}$ drawn from the following ten-bit strings:

$\begin{array}{llll}0000000000 & 1100101101 & 1100000100 & 0010010010 \\ 1001100100 & 0111011011 & 1101111110 & 0010111011 \\ 1001101111 & 0110110000 & 1111000101 & 1011010100 \\ 0101100000 & 1011011111 & 0101101011 & 0011000001 \\ 0000101001 & 1110010110 & 0001111010 & 1110111111\end{array}$

\begin{tabular}{|l|r|r|r|r|}
\hline $\mathrm{n} \backslash \mathrm{d}$ & 2 & 3 & 4 & 5 \\
\hline 4 & 4 & - & - & - \\
\hline 5 & 6 & 2 & - & - \\
\hline 6 & 16 & 2 & 1 & - \\
\hline 7 & $24-26$ & $2-3$ & $0-1$ & - \\
\hline 8 & 32 & $8-9$ & 1 & - \\
\hline 9 & $96-112$ & $\mathbf{1 2 - 1 3}$ & 1 & - \\
\hline 10 & 256 & $\mathbf{2 0}-24$ & $4-5$ & 1 \\
\hline 11 & $386-460$ & $\mathbf{4 8}-53$ & $4-7$ & 2 \\
\hline 12 & $2^{10}$ & $64-89$ & $16-20$ & 2 \\
\hline
\end{tabular}

Fig. 2. Upper and lower bounds on the optimal $K$ for a nonadditive $((n, K, d))$ code. The lower bounds are drawn from [7], [8], [9], [10], [19] as well as the new constructions presented here. The upper bounds are obtained from the linear program of [18], and for distance 2 its improvement in [19]. The new lower bounds from [10] and this work are in bold.

\section{DISCUSSION}

We have presented a new framework for quantum codes and shown how it encompasses stabilizer codes, elucidates the structure of the known nonadditive codes, as well as generates new nonadditive codes with excellent performance. Our codeword stabilized codes are described by two objects: First, the codeword stabilizer that without loss of generality can be taken to describe a graph state, and which transforms the quantum errors to be corrected into effectively classical errors. And second, a classical code capable of correcting the induced classical error model. With a fixed stabilizer state, finding a quantum code is reduced to finding a classical code that corrects the (perhaps rather exotic) induced error model. Our new codes help further fill in the the table of lower bounds on known codes (see Fig. 27. We also show that CWS codes include all stabilizer codes. This new way of thinking of stabilizer codes may help to find new codes with good properties.

In a future work we hope to expand our work in several new areas. We will give algorithms for finding codes (some of which were employed to find the new codes presented here) as well as bounds on the computational complexity of the algorithms. We also will study the circuits for implementing CWS codes and their transversal properties which need to be understood if they are to be useful for fault-tolerant quantum computation. Finally, we hope to find more new codes, especially of distance higher than three.

\section{REFERENCES}

[1] P. Shor, "Algorithms for quantum computation: discrete logarithms and factoring," Foundations of Computer Science, pp. 124-134, 1994.

[2] R. Feynman, "Simulating physics with computers," Internation Journal of Theoretical Physics, vol. 21, pp. 467-488, 1982.

[3] - "Quantum mechanical computers," Foundations of Physics, vol. 16 , no. 6, pp. 507-531, 1986.

[4] S. Lloyd, "Universal quantum simulators," Science, no. 273, p. 1073, 1996.

[5] D. Aharonov and M. Ben-Or, "Fault-tolerant quantum computation with constant error," Proceedings of the twenty-ninth anпиаl ACM symposium on Theory of computing, pp. 176-188, 1997.

[6] D. Gottesman, "Stabilizer codes and quantum error correction," Caltech Ph.D. Thesis.

[7] A. R. Calderbank, E. M. Rains, P. W. Shor, and N. Sloane, "Quantum error correction via codes over gf(4)," IEEE Trans. Inf. Theory, vol. 44, pp. 1369,1387, 1998, arXiv quant-ph/9608006

[8] E. M. Rains, R. H. Hardin, P. W. Shor, and N. J. A. Sloane, "A nonadditive quantum code," Phys. Rev. Lett., vol. 79, pp. 953-954, 1997, arXiv quant-ph/9703002

[9] J. A. Smolin, G. Smith, and S. Wehner, "A simple family of nonadditive quantum codes," arXiv quant-ph/0701065

[10] S. Yu, Q. Chen, C. H. Lai, and C. H. Oh, "Nonadditive quantum errorcorrecting code," arXiv:quant-ph/0704.2122.

[11] V. Aggarwal and A. R. Calderbank, "Boolean functions, projections operators and quantum error correcting codes," arXiv/cs/0610159

[12] V. Arvind, P. Kurur, and K. Parthasarathy, "Nonstabilizer quantum codes from abelian subgroups of the error group," arXiv quant-ph/0210097

[13] C. H. Bennett, D. P. DiVincenzo, J. A. Smolin, and W. K. Wootters, "Mixed state entanglement and quantum error correction," Phys.Rev. A., vol. 54, pp. 3824-3851, 1996, arXiv quant-ph/9604024

[14] E. Knill and R. Laflamme, "Theory of quantum error-correcting codes," Phys. Rev. A, vol. 55, no. 2, pp. 900-911, 1997.

[15] D. Schlingermann, "Stabilizer codes can be realized as graph codes," Quantum Information \& Computation, vol. 2, no. 4, pp. 307-323, 2002, arXiv quant-ph/0111080

[16] M. Grassl, A. Klappenecker, and M. Rötteler, "Graphs, quadratic form, and quantum codes," 2002 IEEE International Symposium on Information Theory (ISIT), p. 45, 2002.

[17] M. Van den Nest, J. Dehaene, and B. DE Moor, "Graphical description of the action of local clifford transformations on graph states," Phys. Rev. A., vol. 69, no. 022316, 2004.

[18] E. Rains, "Quantum shadow enumerators," IEEE Trans. Inf. Theory, vol. 45, no. 7, pp. 2361-2366, 1999, arXiv quant-ph/9611001

[19] - "Quantum codes of minimum distance two," IEEE Trans. Inf. Theory, vol. 45, no. 1, pp. 266-271, 1999, arXiv quant-ph/9704043 